\title{
Compaction-Based Deformable Terrain Model as an Interface for Real-Time Vehicle Dynamics Simulations
}

\begin{abstract}
This paper discusses the development of a deformable terrain database to be used in a co-simulation environment with a multibody dynamics vehicle model. The implementation of the model includes a general tire-terrain traction model which is modular to allow for any type of tire model that supports the Standard Tire Interface[1] to operate on the terrain. Rather than utilizing empirical terramechanics models that only consider the pressure/sinkage directly under the tire, the governing equations of the terrain are based on a soil compaction model that includes both the propagation of subsoil stresses due to vehicular loads, and the resulting visco-elastic-plastic stress/strain on the affected soil volume. Pedo transfer functions allow for the calculation of the soil mechanics model parameters from existing soil measurements. This terrain model was implemented in a way that maps well to Graphics Processor Unit, which allows the model to run in realtime, enabling operator in the loop full vehicle simulations. Test simulations are run using a rigid tire with lugs to show the capability of the model to predict tire and terrain responses. Run times and scaling analyses are presented to gauge the relative speedup of utilizing GPUs for computational acceleration.
\end{abstract}

\section{INTRODUCTION}

In order to enable off-road vehicle dynamics analysis simulations when traveling on soft soil, a deformable Vehicle Terrain Interface (VTI) model that interfaces with existing vehicle and tire dynamics models was developed. It is modularized in such a way that it can be interfaced to a multibody dynamics vehicle that supports the Standard Tire Interface(STI)[1]. Any tire model can be used that supports the STI as well; currently, both rigid[2] and flexible[3] tire models are supported. A detailed description of the model as an API is given in section 1.

Geometry associated with the terrain profile is a combination of low-fidelity terrain height measurements, e.g., OpenFlight terrain database format, with superimposed NURBS for high-frequency content[4]; an example terrain profile is shown in Figure 1. The terrain model is able to efficiently query the current height by locally defining the surface as an equidistantly spaced $\mathrm{x}$-y grid, and using bi-linear interpolation of the nearest four points. Points on the surface also contain information on the state of deformation of the soil, notably the undisturbed terrain height, the type of terrain, e.g. USCS soil classification code, and the energy and power involved in the soil deformation [5]. The terrain takes a set of tire-terrain interface forces from the tire model (Figure 2), applies it to the surface of the terrain, evaluates the terramechanics problem and updates the soil states and deformed surface profile for the subsequent time step.

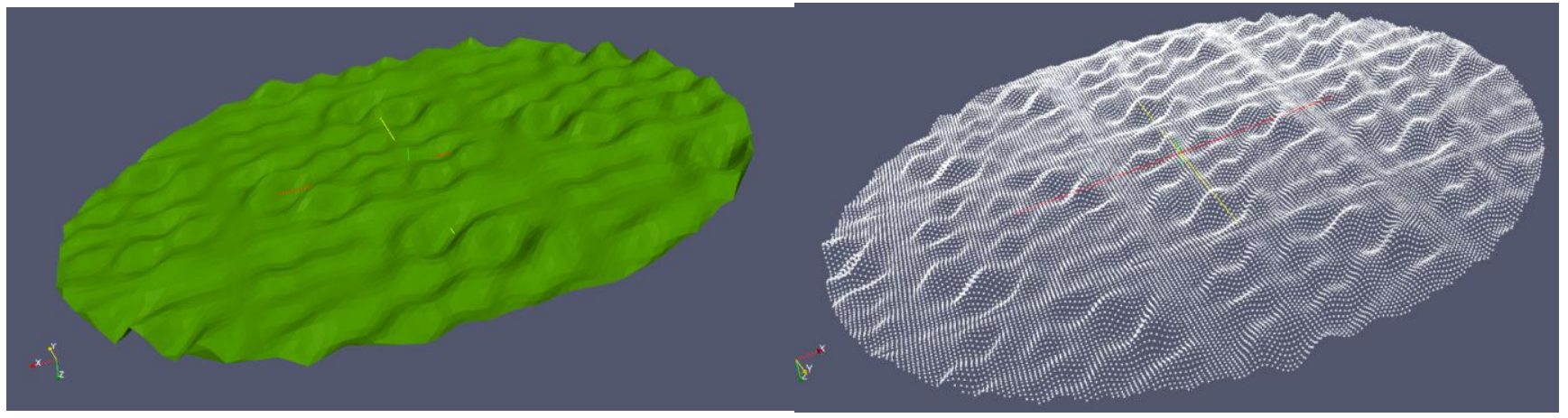

Figure 1. Terrain geometry superimposes low and high-frequency content; shown as a surface (left) and as the true heightmap (right) 


\begin{tabular}{|c|c|c|c|c|c|}
\hline \multicolumn{4}{|c|}{ Report Documentation Page } & \multicolumn{2}{|c|}{$\begin{array}{l}\text { Form Approved } \\
\text { OMB No. 0704-0188 }\end{array}$} \\
\hline \multicolumn{6}{|c|}{ 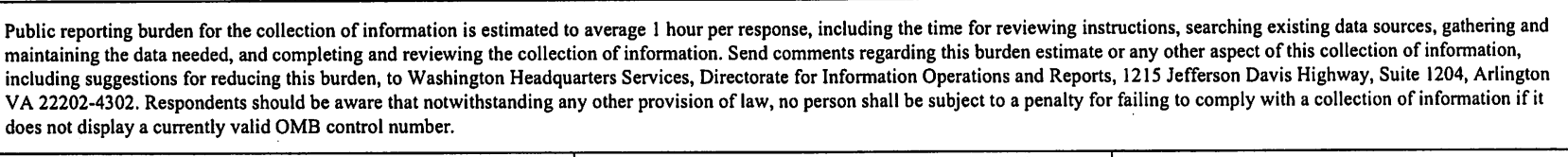 } \\
\hline \multicolumn{2}{|l|}{$\begin{array}{l}\text { 1. REPORT DATE } \\
16 \text { APR } 2013\end{array}$} & \multicolumn{2}{|l|}{ 2. REPORT TYPE } & \multicolumn{2}{|c|}{ 3. DATES COVERED } \\
\hline \multirow{3}{*}{\multicolumn{4}{|c|}{$\begin{array}{l}\text { 4. TITLE AND SUBTITLE } \\
\text { Compaction-Based Deformable Terrain Model as an Interface for } \\
\text { Real-Time Vehicle Dynamics Simulations }\end{array}$}} & \multicolumn{2}{|c|}{ 5a. CONTRACT NUMBER } \\
\hline & & & & \multicolumn{2}{|c|}{ 5b. GRANT NUMBER } \\
\hline & & & & \multicolumn{2}{|c|}{ 5c. PROGRAM ELEMENT NUMBER } \\
\hline \multirow{3}{*}{\multicolumn{4}{|c|}{$\begin{array}{l}\text { 6. AUTHOR(S) } \\
\text { Alexander Reid }\end{array}$}} & \multicolumn{2}{|c|}{ 5d. PROJECT NUMBER } \\
\hline & & & & \multicolumn{2}{|c|}{ 5e. TASK NUMBER } \\
\hline & & & & \multicolumn{2}{|c|}{ 5f. WORK UNIT NUMBER } \\
\hline \multicolumn{4}{|c|}{$\begin{array}{l}\text { 7. PERFORMING ORGANIZATION NAME(S) AND ADDRESS(ES) } \\
\text { U.S. Army TARDEC,6501 East Eleven Mile Rd,Warren,Mi,48397-5000 }\end{array}$} & \multicolumn{2}{|c|}{$\begin{array}{l}\text { 8. PERFORMING ORGANIZATION } \\
\text { REPORT NUMBER } \\
\# 23427\end{array}$} \\
\hline \multirow{2}{*}{\multicolumn{4}{|c|}{ 9. SPONSORING/MONITORING AGENCY NAME(S) AND ADDRESS(ES) }} & \multicolumn{2}{|c|}{ 10. SPONSOR/MONITOR'S ACRONYM(S) } \\
\hline & & & & \multicolumn{2}{|c|}{$\begin{array}{l}\text { 11. SPONSOR/MONITOR'S REPORT } \\
\text { NUMBER(S) }\end{array}$} \\
\hline \multicolumn{6}{|c|}{$\begin{array}{l}\text { 12. DISTRIBUTION/AVAILABILITY STATEMENT } \\
\text { Approved for public release; distribution unlimited. }\end{array}$} \\
\hline \multicolumn{6}{|c|}{ 13. SUPPLEMENTARY NOTES } \\
\hline \multicolumn{6}{|c|}{$\begin{array}{l}\text { 14. ABSTRACT } \\
\text { This paper discusses the development of a deformable terrain database to be used in a co-simulation } \\
\text { environment with a multibody dynamics vehicle model. The implementation of the model includes a } \\
\text { general tire-terrain traction model which is modular to allow for any type of tire model that supports the } \\
\text { Standard Tire Interface[1] to operate on the terrain. Rather than utilizing empirical terramechanics } \\
\text { models that only consider the pressure/sinkage directly under the tire, the governing equations of the } \\
\text { terrain are based on a soil compaction model that includes both the propagation of subsoil stresses due to } \\
\text { vehicular loads, and the resulting visco-elastic-plastic stress/strain on the affected soil volume. Pedo } \\
\text { transfer functions allow for the calculation of the soil mechanics model parameters from existing soil } \\
\text { measurements. This terrain model was implemented in a way that maps well to Graphics Processor Unit, } \\
\text { which allows the model to run in realtime, enabling operator in the loop full vehicle simulations. Test } \\
\text { simulations are run using a rigid tire with lugs to show the capability of the model to predict tire and } \\
\text { terrain responses. Run times and scaling analyses are presented to gauge the relative speedup of utilizing } \\
\text { GPUs for computational acceleration. }\end{array}$} \\
\hline \multicolumn{6}{|l|}{ 15. SUBJECT TERMS } \\
\hline \multicolumn{3}{|c|}{ 16. SECURITY CLASSIFICATION OF: } & 17. LIMITATION OF & 18. NUMBER & \\
\hline $\begin{array}{c}\text { a. REPORT } \\
\text { unclassified }\end{array}$ & $\begin{array}{c}\text { b. ABSTRACT } \\
\text { unclassified }\end{array}$ & $\begin{array}{l}\text { c. THIS PAGE } \\
\text { unclassified }\end{array}$ & & $\begin{array}{c}\text { OF PAGES } \\
8\end{array}$ & RESPONSIBLE PERSON \\
\hline
\end{tabular}




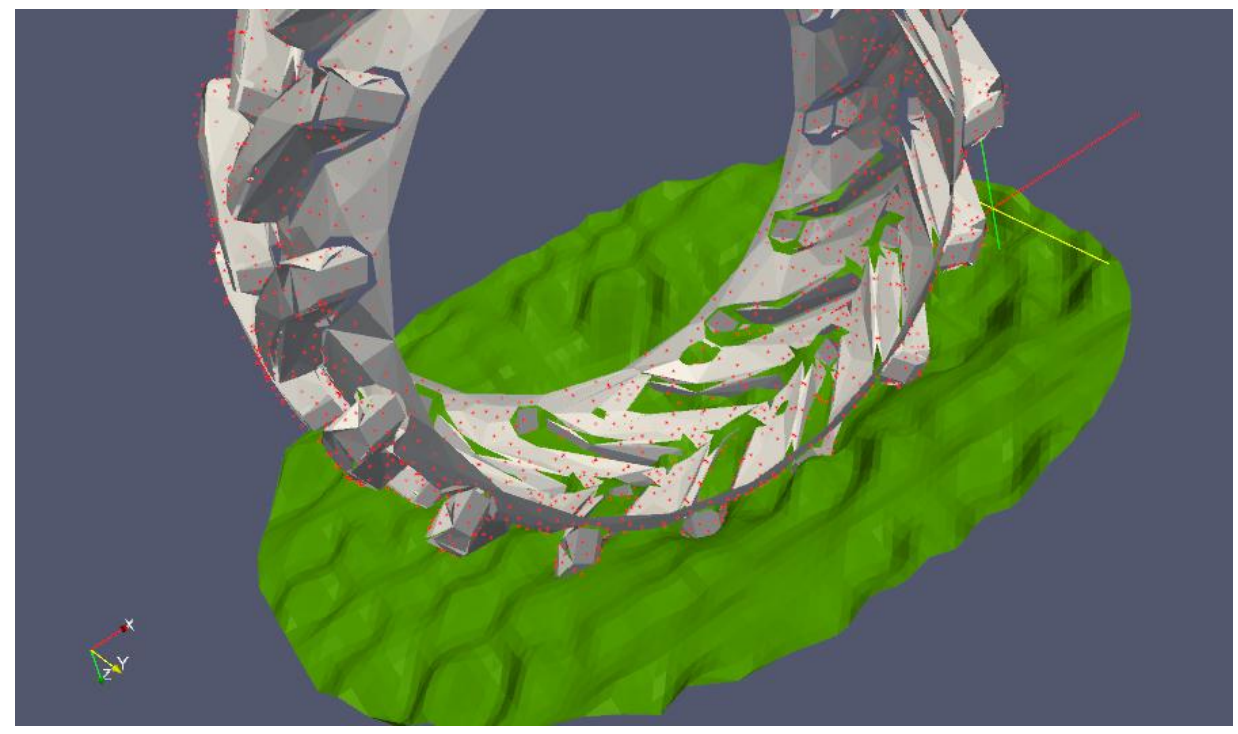

Figure 2. Tire geometry used to determine collision points with the terrain

In the context of off-road vehicle simulations, terrain models fall into three categories of increasing complexity: rigid terrain where the main focus is an accurate surface profile [6-9], use of empirical relationships to find pressure and sinkage directly under the tire [10-12], or finite/discrete element approaches [13-16]. Any off-road vehicle dynamics simulation where the soil deforms considerably requires a terrain model that accurately reflects the deformation and response of the soil to all possible inputs of the tire in order to correctly simulate the response of the vehicle[17]. Typical vehicle dynamics simulations for mobility or component fatigue analysis purposes require many simulation runs with time-lengths in the $10 \mathrm{~s}$ to $100 \mathrm{~s}$ second range, which makes complex finite/discrete element approaches unfeasible. Purely empirical terrain models also have considerable drawbacks: the parameters are sensitive to the size and shape of the test plate[18], they do not scale to vehicles with small contact patches[19], and only consider the sinkage directly under the applied surface load[20]. Furthermore, the time-dependent elastic and plastic response of the soil to repetitive compression/rebound tire loads cannot be taken into account[10]. The goal of the terrain mechanics model is to be as realistic as possible by tracking the stress and strain of the soil volumetrically by utilizing a vertical soil compaction model. A major constraint on the model is imposed by the requirement of running at realtime speeds to enable operator in the loop simulations. The theoretical framework of the soil mechanics model has been previously discussed [2].

In the proposed approach fast simulations will be possible due to the inherent parallel nature of the subsoil stress calculations which are the major computational bottlenecks. Parallel CPU and GPU hardware is utilized to accelerate these computations, and details of the implementation strategy are given in section 2. Example simulations will include timing and profiling results for sequential, CPU parallel and CPU \& GPU parallel implementations in section 3.

The model will be exercised using a rigid tire with and without lugs to demonstrate the ability to handle complicated tire geometry. Simulation experiments are detailed in section 3. Results will include the computed tire-terrain contact forces developed by the rigid tire, as well as the deformed state of the terrain.

\section{Vehicle Tire Terrain Interaction Model Components}

The Vehicle-Terrain Interaction model involves three main components: (i) a surface loading mechanism due to 3D tire geometry contact with the terrain, as shown in Figure 2, (ii) stress propagation of the load through the subsoil, and (iii) rigorous vertical soil stress/strain relationship. Calculation flow can be visualized as shown in Figure 3 to help understand the dependencies of each part of the model.

At the beginning of a time step, the vehicle passes the tire wheel spindle state data, which includes the position and velocity of the Center of Mass (CM) in the reference frame indicated by the Standard Tire Interface[1]. The tire passes an updated geometry profile to the terrain in the form of a heightmap query, and the terrain database returns the collision information. Forces at the tire/terrain interface are found at each time step by using a combination of normal and slip forces, in conjunction with soft-soil tire boundary forces, e.g. bulldozing and slip-sinkage effects. These forces are passed from the tire to the terrain model, where the terrain model applies Boussinesq and Cerruti soil mechanics equations to determine the pressure distribution in the volume of affected

Page 2 of 8 
material. The model treats a column of soil as a system of discretized soil volumes, and each volume element is modeled using viscoelasto-plastic compressibility relationships that relate subsoil pressures to a change in bulk density of the soil, which in turn produces soil displacements and changes to other soil state variables. Different loading combinations applied by a tire passing over a column of soil will be reflected in the state of each volume of soil contained in the column, rather than treating the column of soil as homogeneous in the vertical direction and only associating one set of parameters with the entire column. The outputs of the terrain model include: tire-terrain pressure distribution, terrain surface deformation, updated soil states and power/energy required to deform the soil.

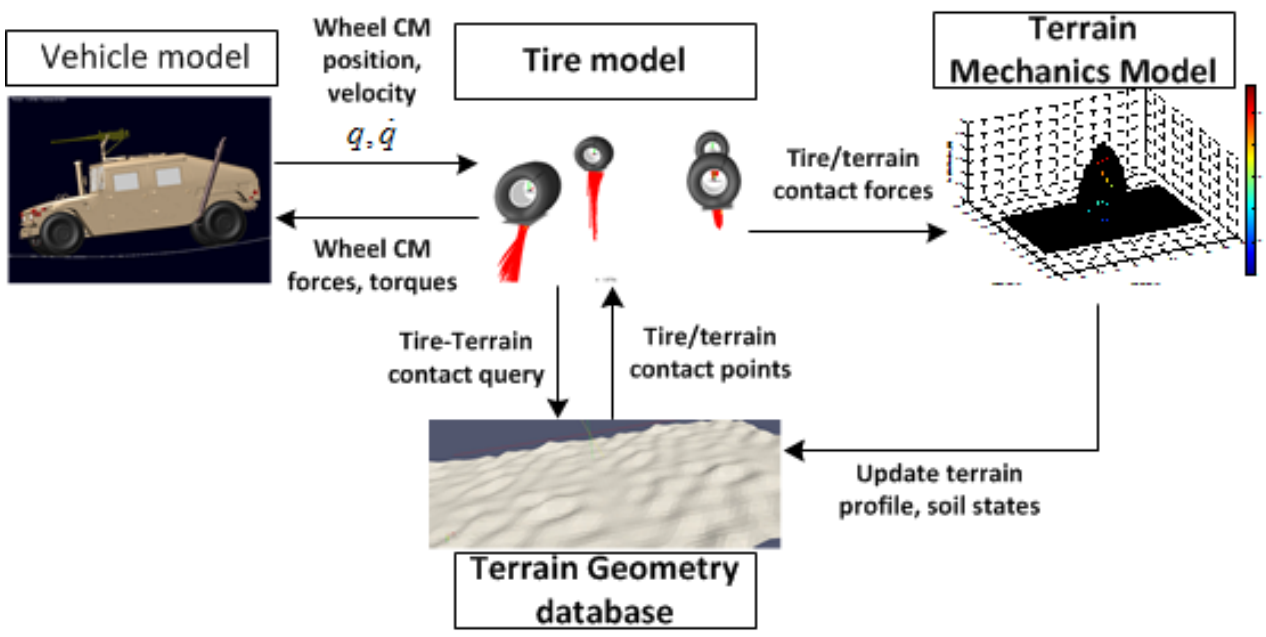

Figure 3. Flow chart of the VTI functionality as an extension of the STI

\section{Terrain Model Acceleration with GPU computing}

There currently is a trend in advanced scientific computing to offload repetitive calculations to accelerators/coprocessors, such as Graphics Processing Units (GPUs). These accelerators consist of many lightweight processors which allow them to perform multiple basic calculations at the same time. Nvidia's current Kepler family of GPUs can have up to 1536 of these lightweight processors, thereby allowing these GPUs to perform calculations on up to 1536 pieces of data at the exact same time. Furthermore GPUs are designed to have a low cost for context switching, meaning that the GPU can quickly switch between threads of calculations if a thread is temporarily stalled (such as is the case when waiting to load data from global memory). These properties of a large number of processing elements with low cost context switching, combined with a high speed memory bandwidth, allow GPUs to efficiently perform the same calculations on tens of thousands of pieces of data.

In general, the subsoil stress calculations need to be performed on each point in the soil volume being analyzed, for each contact force point on the soil due to the applied load. For a typical simulation with a 1"x1"x1" grid resolution, there can be approximately 56000 subsoil volumes with 400 contact forces to be considered. Since the calculations performed for each subsoil volume are exactly the same (albeit with different data), the GPU is a good option to help accelerate the simulation. This was done using Nvidia's CUDA framework for calculations and the Thrust library for memory management. For the sake of comparison, a CPU version of the subsoil stress calculations was implemented using OpenMP.

A comparison of the total runtimes for the CPU and GPU implementations for a five second simulation with a 50ms timestep for grid resolutions ranging from 0.5 " 0.5 " $\mathrm{x} 1$ " to 3 " $\mathrm{x} 3$ " $\mathrm{x} 1$ " is shown in Figure 4. The CPU simulations were performed on a machine with four 16 core AMD Opteron 6274 processors, allowing for 64 simultaneous threads. The GPU simulations were performed on a machine with an Nvidia Tesla C2070 and two six core Intel Xeon E5-2630 processors. It can clearly be seen that the GPU is faster than the CPU for finer resolution grids - those with a larger number of subsoil volumes. 


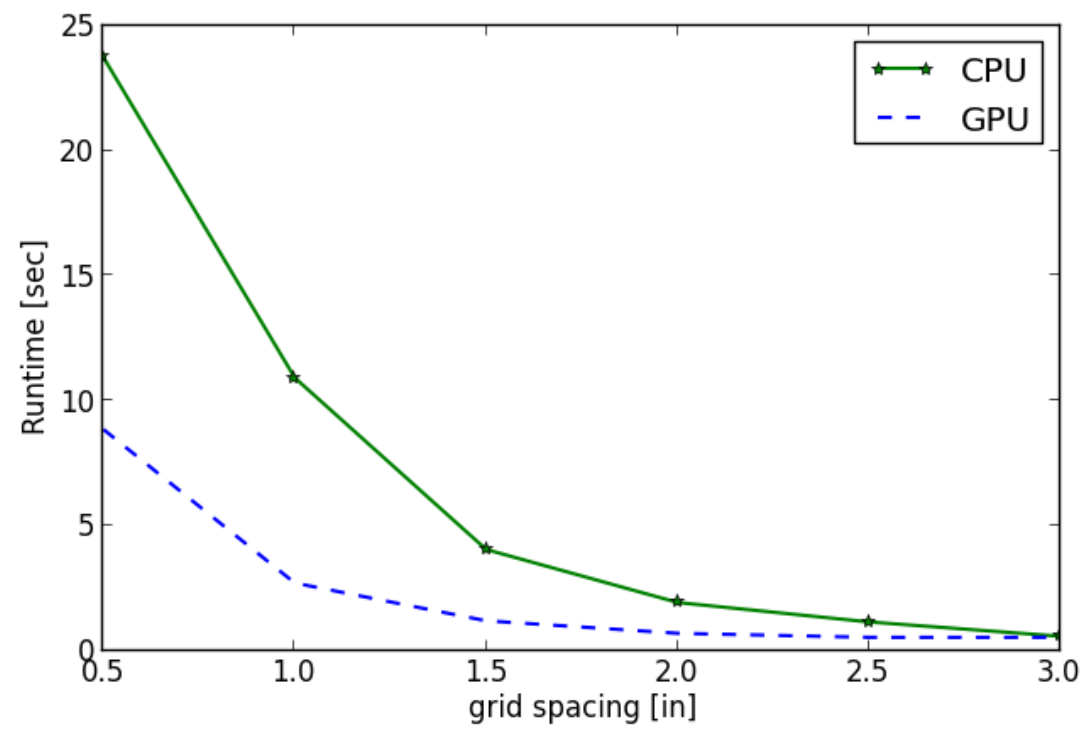

Figure 4. Total runtime for a 5 second single tire simulation, CPU and GPU, as a scaling analysis

The runtime required for a simulation is roughly proportional to the product of the number of subsoil volumes and tire contact points (essentially, the total number of stress calculations performed). It can clearly be seen that the GPU is approximately 8-9x faster than the CPU, on average. Given that the previous figure shows the GPU to only be about $4 \mathrm{x}$ faster for the entire simulation at the given resolution (1"x1"x1"), means that the GPU implementation has reached a point where the overhead of the rest of the simulation code is taking up more time than the subsoil stress calculations. It should be noted that timings reported here include the overhead of moving the subsoil node data to and from the GPU.

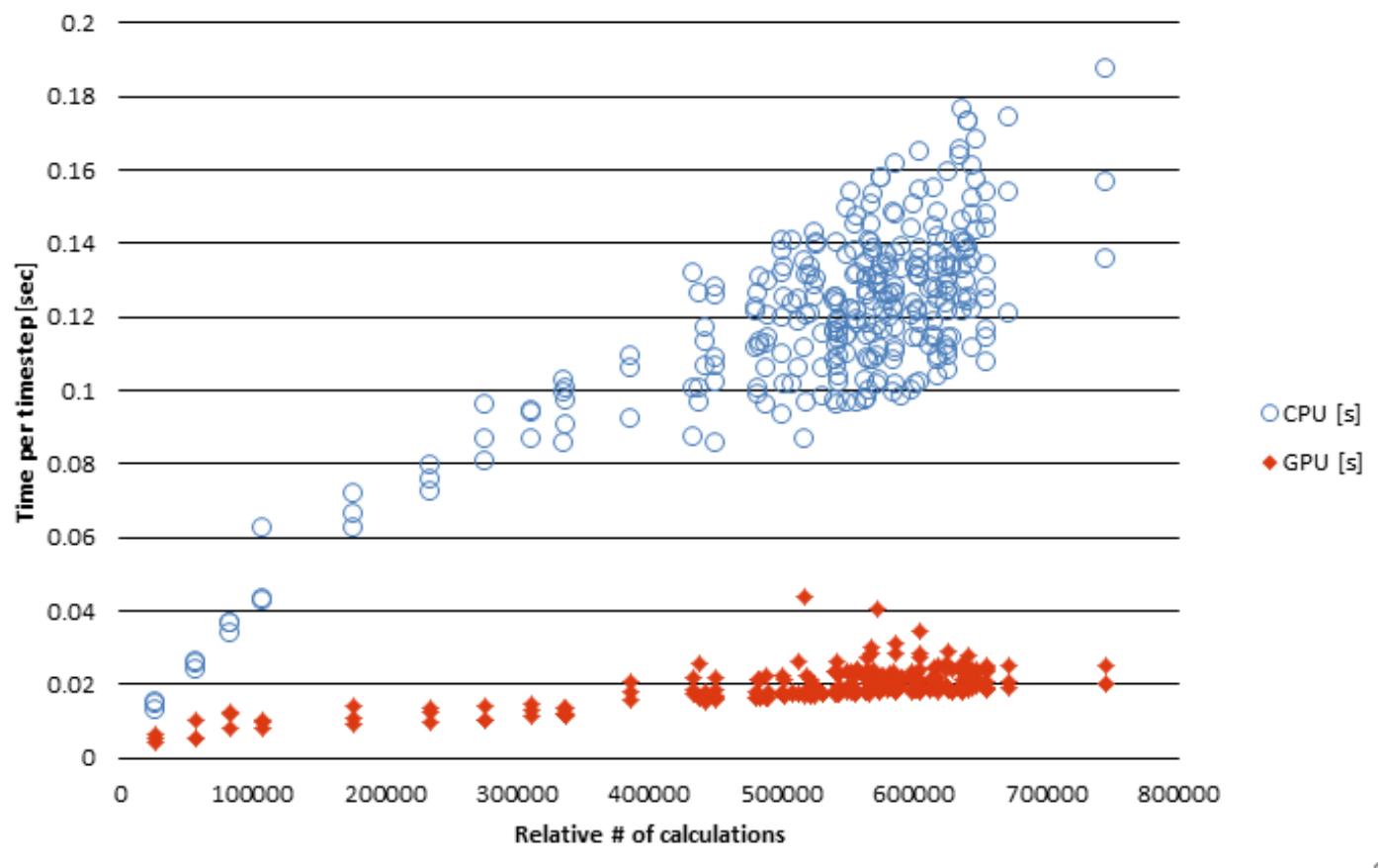

Figure 5. Time per step v. relative number of calculations per step

\section{Numerical Results}

In order to determine the effect the subsoil stress equations have when they are applied to the discretized soil volume, a simplified tire that is assumed to be rigid applies a normal force on the surface of the terrain according to the tire-terrain interaction Page 4 of 8 
model. The following settings were used for the terrain mechanics settings of all soil nodes: an assumed Frolich stress concentration parameter of 4 , an initial bulk density of $0.04515911\left[\mathrm{lb} / \mathrm{in}^{3}\right]$, preconsolidation stress, $\sigma_{\mathrm{k}}=10.356$ [psi], with a CL soil type. A compression/rebound soil response is caused by an applied vertical displacement of 7 "to the tire over 1 second, followed a horizontal wheel displacement at a constant velocity to demonstrate the rigid wheel's effects on the terrain while traveling at a steady state velocity of 1.5 MPH. A rotational displacement is applied to result in a minimal value of slip. The deformed and undeformed terrain surface profiles are shown in Figure 6, and the tire-terrain interaction forces during the final timestep are shown in Figure 7. The deformation energy for both the current step and cumulatively are shown in Figure 8.

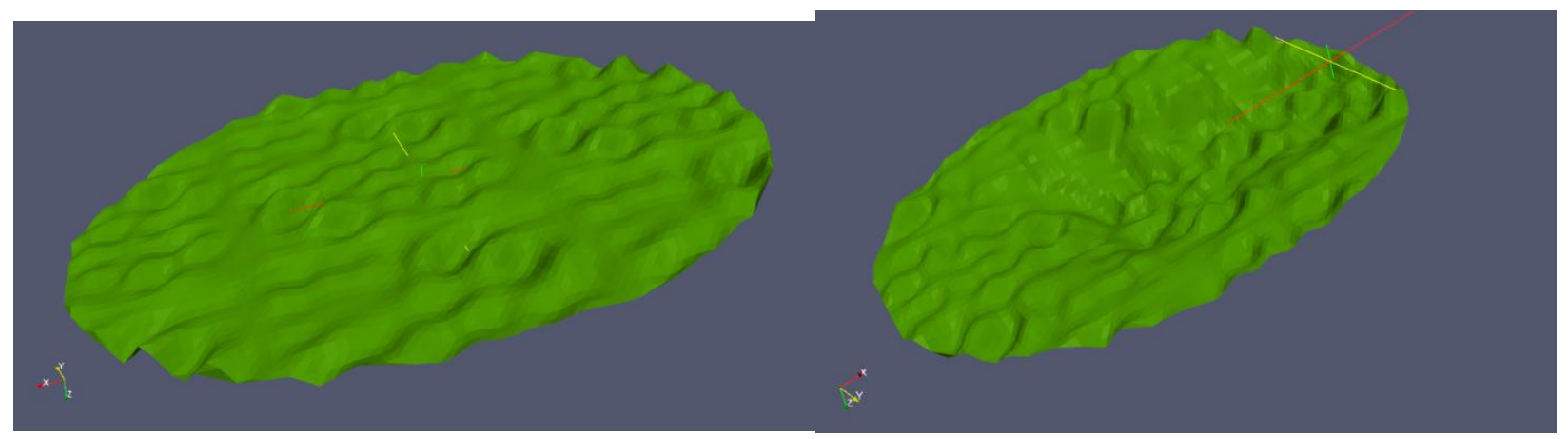

Figure 6. Undeformed (left) and Deformed (right) Terrain surface profiles

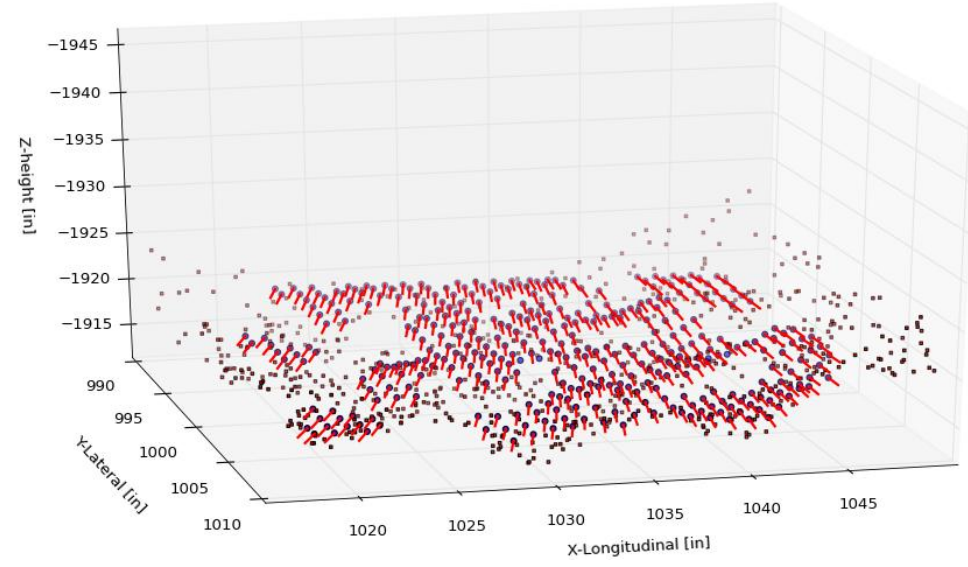

Figure 7. Tire-Terrain interaction forces, low slip simulation, final timestep
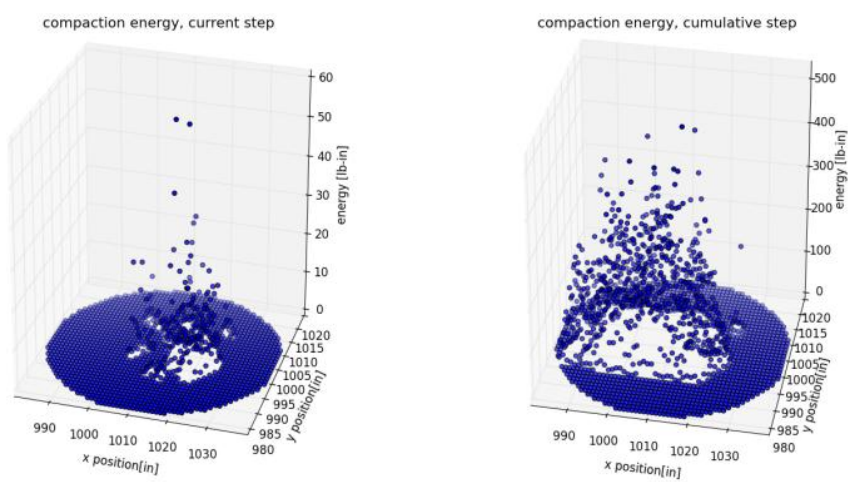

Figure 8. Total and current deformation energy, low slip simulation, final step 
The second simulation example is run, which is similar to the first, except that the rotational displacement is applied to give the tire a slip rate of approximately $15 \%$. As shown by Figure 9, the total displacement of the soil in the vertical direction at the end of the simulation is concentrated about the loading area, i.e. the contact patch, and is strongly influenced by the choice of Frolich parameter value. The total energy required to cause the deflection in Figure 9 is shown in Figure 11, and similar to the total deflection is concentrated about the loading axis. The total energy is a strong function of the maximum soil bulk density, as shown by the large peaks in Figure 11. Tire-terrain interaction forces in the final step of the simulation are shown in Figure 10, and it can be seen that there are slightly larger force vectors when compared to the low slip simulation, Figure 7 (mainly elongated in the direction of travel) indicating that the shear displacement-shear stress relations properly account for the tractive forces induced by wheel slip.

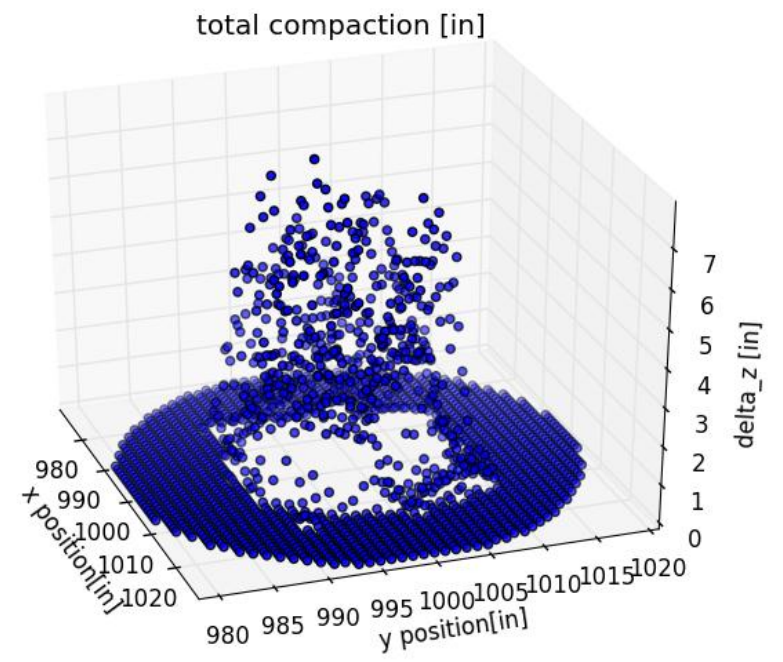

Figure 9. Total vertical displacement of the terrain, $15 \%$ slip simulation

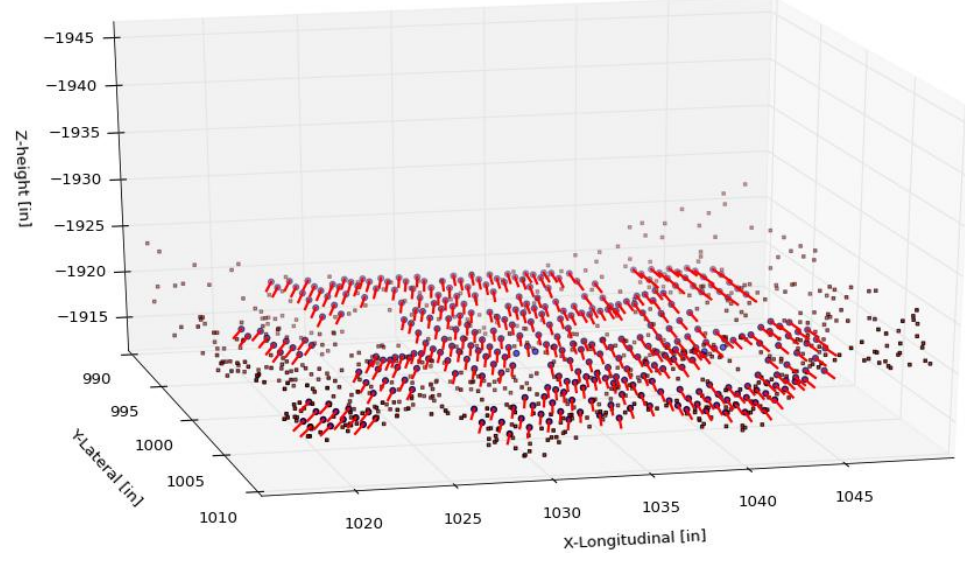

Figure 10. Tire-Terrain interaction forces, $15 \%$ slip simulation, final timestep 

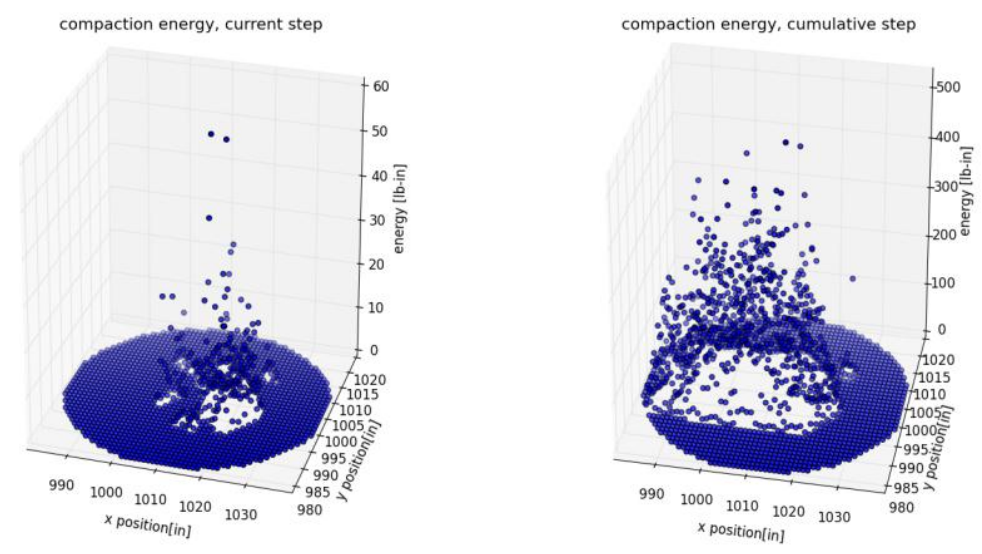

Figure 11. Total deformation energy after 15\% slip simulation

\section{Conclusion}

The development of a general 3D tire-terrain traction model which operates on a novel deformable terrain representation that utilizes a soil compaction model is presented and discussed. The tire and vehicle models are assumed to be contained in commercial software, and are linked to the terrain model using an API based on the Standard Tire Interface. The soil model is an improvement to what is typically found in commercial vehicle dynamics packages, as it considers the subsoil stress propagation as well as the soil stress-strain relationship. It also captures the change in soil state in a volumetric sense, and does not make the assumption that the terrain remains homogenous in the vertical direction, which is what is typically done in most empirical terramechanics codes. Repeated loading and time-domain effects are taken into account, and the change in soil bulk density can be utilized to calculate the required energy and power to deform the terrain. It is predicted that this model will result in not only more realistic mobility simulations, but also allow for a novel power and energy analysis technique to predict the additional power required to operate a vehicle on deformable terrain.

Example numerical results verify that the terrain database can handle tire geometry that is complex and non-uniform. The terrain model leverages parallel computing using both CPUs and GPUs and is shown to scale well, which will enable real-time deformable terrain to be simulated. This paper represents an ongoing effort between the University of Tennessee, University of Wisconsin-Madison, Mechsim, and the US Army TARDEC to incorporate the physics-based VTI model into a real-time, operator inthe-loop ride-motion vehicle simulator. Validation of the simulation results with experimental measurements taken at ERDC is currently underway and refinements in various parts of the terrain model are expected.

\section{REFERENCES}

[1] Riedel, A., 1996, "Standard Tire Interface, Issued by TYDEXWorking Group."

[2] Madsen, J., Seidl, A., Negrut, D., Ayers, P., Bozdech, G., Freeman, J., O'Kins, J., and Reid, A., 2011, "A Physics-Based Vehicle/Terrain Interaction Model for Soft Soil Off-Road Vehicle Simulations," SAE World Congress, SAE, Detroit, MI, p. Submitted.

[3] Negrut, D., and Freeman, J., 1994, "Dynamic Tire Modelling for Application with Vehicle Simulations Incorporating Terrain," SAE International Congress \& Expo, SAE, Detroit, MI.

[4] Morrison, M., Romano, R., Reid, A., and Gorsich, D., 2004, "High-Frequency Terrain Content and Surface Interactions for OffRoad Simulations," SAE.

[5] Madsen, J., Seidl, A., Ayers, P., Bozdech, G., Negrut, D., and O'Kins, J., 2012, "A Physics-Based Terrain Model for Off-Road Vehicle Simulations," IDETC/CIE, ASME, ed.Chicago, IL.

[6] Yoon, S., 2003, "A Study on Terrain-Surface Modeling and Searing Algorithms for Real-time Simulation of Off-road Vehicles," Vehicle System Dynamics, 39(5), pp. 353-363.

[7] Gipser, M., 2008, "RGR Road Models for FTire."

Page 7 of 8 
[8] Wagner, S., and Ferris, J., 2009, "Developing stable autoregressive models of terrain topology," Inter. J. of Veh. Systems Modelling and Testing, 4(4), pp. 306-317.

[9] Detweiler, Z., and Ferris, J., 2010, "Interpolation Methods for High-Fidelity Three Dimensional Terrain Surfaces," J. of Terramechanics, 47(4), pp. 209-217.

[10] Bekker, M. G., 1956, Theory of land locomotion; the mechanics of vehicle mobility, University of Michigan Press, Ann Arbor,.

[11] Wong, J. Y., and ScienceDirect (Online service), 2010, "Terramechanics and off-road vehicle engineering terrain behaviour, offroad vehicle performance and design," Elsevier, Amsterdam, pp. 1 online resource (xix, 463 p.).

[12] Reece, A. R., "Principles of Soil-Vehicle Mechanics," Proc. Vol. 180, Part 2A, The Institution of Mech. Engrs.

[13] Shmulevich, I., 2010, "State of the art modeling of soil-tillage interaction using discrete element method," Soil and Tillage Research, 111(1), pp. 41-53.

[14] Perumpral, J. V., Liljedahl, J. B., and Perloff, W. H., 1971, "A numerical method for predicting the stress distribution and soil deformation under a tractor wheel," Journal of Terramechanics, 8(1), pp. 9-22.

[15] Horn, R., Richards, B. G., Gräsle, W., Baumgartl, T., and Wiermann, C., 1998, "Theoretical principles for modelling soil strength and wheeling effects - a review —," Zeitschrift für Pflanzenernährung und Bodenkunde, 161(4), pp. 333 -346.

[16] Shoop, S. A., 2001, "Finite Element Modeling of Tire-Terrain Interaction," PhD Thesis, University of Michigan.

[17] Gipser, M., 2007, "FTire - the tire simulation model for all applications related to vehicle dynamics," Vehicle System Dynamics, 45(139-151).

[18] Bekker, M. G., 1960, Off-the-road locomotion; research and development in terramechanics, University of Michigan Press, Ann Arbor,.

[19] Wong, J. Y., 1993, Theory of ground vehicles, J. Wiley, New York, N.Y.

[20] Wulfsohn, D., and Upadhyaya, S. K., 1992, "Prediction of traction and soil compaction using three-dimensional soil-tyre contact profile," Journal of Terramechanics, 29(6), pp. 541-564. 